\title{
Effect of Post-Natal Catch-Down and Feeding Practices on Auxology, Body Composition and Muscle Function in Children Born Large-For- Gestational-Age
}

\author{
Julia Trachternach Peters ${ }^{1}$, Joachim Woelfle ${ }^{1}$, Susanne Joergens ${ }^{1}$, Felix Schreiner ${ }^{1}$, Peter Bartmann ${ }^{2}$ and Bettina Gohlke ${ }^{1^{*}}$ \\ ${ }^{1}$ Department of Pediatric Endocrinology and Diabetology, University Hospital Bonn, Germany \\ ${ }^{2}$ Department of Neonatology, University Hospital Bonn, Germany \\ "Corresponding author: Prof. Dr. med. Bettina Gohlke, Department of Pediatric Endocrinology and Diabetology, University Hospital Bonn, Adenauerallee 119,53113 \\ Bonn, Germany, Tel: +49-22828733262; Fax: + 49228287 33344; E-mail: Bettina.gohlke@ukb.uni-bonn.de
}

Received date: August 18, 2017; Accepted date: September 08, 2017; Published date: September 15, 2017

Copyright: (C) 2017 Peters JT, et al. This is an open-access article distributed under the terms of the Creative Commons Attribution License, which permits unrestricted use, distribution, and reproduction in any medium, provided the original author and source are credited.

\begin{abstract}
Background: High birth-weight and supernormal postnatal weight gain increases the risk of obesity. Breastfeeding and postnatal Catch-down growth in children born large-for-gestational-age (LGA) might be protective.

Methods: 101 LGA-children of mothers without gestational diabetes were included; 60 breastfed. Auxology and muscle function were examined at 8.0yrs (range, 4.3-12.1). Data were analysed in relation to birth-weight and/or length, to catch-down growth, and to feeding practices.

Results: A strong familiarity (44\% LGA-siblings; 31 LGA-mothers (38.8\%); 21 LGA-fathers (34.4\%)) and a positive correlation to mother's birth weight-SDS $(r: 0.23, p=0.042)$ and -length $(r: 0.25, p=0.027)$ were found.

Postnatal catch-down growth was found in $88 \%$ (89/101) with no difference regarding to feeding practices. At school age mean BMI-SDS was normal but height-SDS remained significantly higher than target height-SDS. Those $12 \%$ without postnatal catch-down were significantly taller and heavier, had higher skinfold-SDS and waistcircumference-SDS than those with catch-down growth- irrespective of feeding practice. Muscle function was below average in formerly heavy but normal in those solely long LGA-infants.

Conclusion: Irrespective of feeding practice the majority of LGA-children showed catch-down growth. However catch-down growth did not lead to a complete normalization of auxologic parameters. Importantly in those without postnatal catch-down growth higher indices for fatness were observed.
\end{abstract}

Keywords: Anthropometry; Catch-down-growth; LGA; Large-forgestational-age; Muscle function; Breastfeeding

Abbreviations: BMI: Body Mass Index; C-D: Catch-Down; LGA: Large-For-Gestational-Age; $\mathrm{LGA}_{\mathrm{L}}$ : Large for Gestational Age Concerning Birth-Length Only; LGA $\mathrm{W}_{\mathrm{W}}$ Large For Gestational Age; Concerning Birth-Weight Only; $\mathrm{LGA}_{\mathrm{L}+\mathrm{W}}$ : Large for Gestational Age Concerning Birth-Length and -Weight; MIGF: Maximal Isometric Grip Force; PJF: Peak Jump Force; PJP: Peak Jump Power

\section{Introduction}

Birth weight is one of the predictors of neonatal mortality and morbidity. It is well known that children born small for gestational age (SGA) are at an increased risk for obesity and an altered fat distribution in adult life [1] which might lead to insulin resistance and other metabolic and cardiovascular co-morbidities [2-4]. In particular, those SGA infants who experienced a rapid postnatal catch-up growth seem to have an even augmented risk of developing obesity [4].

Less is known about children born large for gestational age (LGA). However, some studies demonstrate that children born LGA have as well an increased risk of developing obesity, insulin resistance, metabolic syndrome, diabetes and early cardiovascular disease [5-9]. Furthermore Toschke et al. [10] showed that rapid weight gain from birth to 2 years was associated with a higher prevalence of overweight at school entry. Though catch-up growth of SGA and supernormal weight gain in infants is associated with obesity and metabolic disturbances it was suggested that catch-down growth in children born LGA could be protective for developing obesity and Taal et al. [11] reported that children born LGA without catch-down growth had more subcutaneous fat mass, higher body mass index (BMI) in childhood and an increased risk for childhood overweight than the majority who showed catch-down growth.

It was assumed that in the last century more babies were born "long and lean", whereas nowadays LGA neonates are more likely to be "long and fat" -and that this would have a different impact on later health [12]. This is in accordance with Hediger et al. [7] who found that the persistent associations of LGA-status with anthropometric indices of body composition in infancy and early childhood were primarily attributable to a variation in the index of muscularity measured by the circumference of mid-upper arm muscle area. Muscle function can be reliable assessed by mechanography. Fricke et al. [13] have shown that jumping force and grip force are both reliable measurements to analyze 
Citation: Peters JT, Woelfle J, Joergens S, Schreiner F, Bartmann P, et al. (2017) Effect of Post-Natal Catch-Down and Feeding Practices on Auxology, Body Composition and Muscle Function in Children Born Large-For-Gestational-Age. J Diabetes Metab 8: 760 . doi: $10.4172 / 2155-6156.1000760$

Page 2 of 6

muscle function. Jumping force as a multi-joint motor movement completes the analysis of grip force as a one joint motor function.

Most studies and meta-analyses [14,15] found that breastfeeding was associated with a significant reduction of the risk of obesity in childhood. However, a recent epidemiologic study by Salahuddin et al. [16] demonstrated that in LGA-infants it is the supernormal birthweight per se and not the feeding practice which can be associated with an increased risk of high BMI-SDS in infancy.

The aim of this study was to analyse the impact of feeding practice (exclusively breastfed vs supplementation of formula) and postnatal catch-down growth on body composition and muscle strength in formerly LGA-infants at school age. In addition, we tested the hypothesis that growth, body composition, and muscle function is different in infants born heavy with LGA due to large birth-weight in comparison to those who presented solely with a birth-length above 95th centile but a birth-weight within normal range.

\section{Methods}

All data were collected from the register of birth between 2000-2007 of the Department of Gynaecology of the University Hospital, Bonn and of the St. Marienhospital, Bonn. By reviewing the birth protocols and medical charts, we collected clinical data including gender, gestational age and auxological birth parameters.

LGA is often defined as a birth weight and/or length that lie above the 90th percentile for that gestational age [17]. However, it has been suggested that the definition be restricted to infants with birth weights greater than 2 standard deviation scores (SDS) above the mean as this more accurately describes infants who are at greatest risk for perinatal morbidity and mortality [18]. We therefore defined large for gestational age as birth weight $\left(\mathrm{LGA}_{\mathrm{W}}\right)$ or length $\left(\mathrm{LGA}_{\mathrm{L}}\right)$ or both $\left(\mathrm{LGA}_{\mathrm{W}+\mathrm{L}}\right)$ when corresponding birth parameters were greater than 2 SDS.

\begin{tabular}{|l|l|}
\hline & $\begin{array}{l}\text { Total } \mathbf{n}=\mathbf{1 0 1} \\
\text { Mean } \mathbf{\pm} \mathbf{S D} \text { ) }\end{array}$ \\
\hline $\begin{array}{l}\text { Age } \\
\text { Sex: } 60 \text { male, } 41 \text { female } \\
\text { n: LGALength: } 27\end{array}$ & \\
LGAweight: 31 & \\
LGALength+Weight: 43 & $8.05 \pm 1.71$ \\
\hline Gestational age (in weeks) & $39.78 \pm 1.25$ \\
Birth-length-SDS & $1.52 \pm 0.85$ \\
Birth-weight-SDS & $1.95 \pm 0.78$ \\
\hline Actual auxological data: & \\
Height-SDS & $0.67 \pm 1.03$ \\
Weight-SDS & $0.51 \pm 0.98$ \\
BMI-SDS & $0.23 \pm 0.92$ \\
Target height-SDS & $0.23 \pm 0.75$ \\
Waist circumference-SDS & $0.4 \pm 1.02$ \\
Hip circumference-SDS & $-0.34 \pm 1.17$ \\
\hline Skin fold Triceps-SDS & $-0.43 \pm 1.64$ \\
Skin fold Subscapular-SDS & $-0.88 \pm 3.66$ \\
Skin folds total-SDS & $-0.43 \pm 1.38$ \\
\hline & \\
\hline
\end{tabular}

Table 1: Descriptive data for the whole group (mean $\pm S D$ ).

Initially 936 LGA-infants were identified. Only Caucasian children of mothers without gestational diabetes $(n=302)$ were included and all were invited to take part. The final cohort of this retrospective study consisted of 101 (33\%) children. All were born after an uneventful pregnancy, Caucasian and pre-pubertal (actual median age: 8.0 years (range, 4.3-12.1 years)). Characteristics of the group are shown in Table 1.

Children had been examined from birth until the age of five in regular intervals by their pediatrician. The actual follow-up examination included physical examination, measurement of anthropometric parameters (height, weight, head-, waist-, and hip circumference), skinfold thickness (subscapular, triceps), maximal isometric grip force (MIGF) [19], mechanographic analyses of motor function, and a questionnaire. MIGF was measured with a hand-held Jamar hydraulic dynamometer. Ground reaction forces were measured by use of the Leonardo Platform ${ }^{\circledR}$ (NovotecGmbH, Pforzheim, Germany) as has described elsewhere $[13,20]$. Motor function was analyzed by goal-directed two-leg jumping. The jump was performed as a counter movement jump and the aim was to jump as high as possible. Three jumps were performed and the one with the highest jump was used for further calculations [13].

The questionnaires contained questions asking the parents for their actual weight, height, birth weight and length, illnesses in their family (high blood pressure, cancer, diabetes, heart attacks), particularities in pregnancy (diabetes, high blood pressure, medications, smoking, drinking), breast-feeding and if siblings or cousins of the subjects were LGA as well.

All data were transformed into SDS. German growth standards were used to calculate SDS [21,22], for waist- and hip SDS northern European growth standards were used [23].

For further analysis the cohort was subdivided into

Concerning anthropometric parameters at birth and

According to catch-down growth

According to feeding practices

Part A:

LGAL, $n=27$, large for gestational age concerning birth-length only

LGAW, $\mathrm{n}=31$, large for gestational age concerning birth-weight only

LGAL+W, n=43, birth-length and -weight $>2$ SDS for their gestational age.

Part B:

Analogue to Ong et al. [4] we defined catch-down growth as a negative change of weight-SDS $>0.67$. Because significant change of growth was observed in the first year of life, time span from birth to 12 months was chosen. According to this definition 12/101 (12\%) did not catch-down (no-cd).

Part C:

b: exclusively breastfeeding for at least 4 months $n=60$

$\mathrm{f}$ : additional or exclusively formular feeding $\mathrm{n}=41$

The Ethics Committee of the University Hospital Bonn reviewed the study. Subjects and parents gave their informed consent.

\section{Statistics}

For statistical analysis we used SPSS 22.0 (IBM Corporation, Armonk, NY, USA). The distributions of quantitative variables were tested for normality using the Kolmogorov-Smirnov test. Data are 
Citation: Peters JT, Woelfle J, Joergens S, Schreiner F, Bartmann P, et al. (2017) Effect of Post-Natal Catch-Down and Feeding Practices on Auxology, Body Composition and Muscle Function in Children Born Large-For-Gestational-Age. J Diabetes Metab 8: 760. doi: $10.4172 / 2155-6156.1000760$

Page 3 of 6

presented as mean and standard deviations unless otherwise stated. Differences in body composition and muscle function parameters among different groups $\left(\mathrm{A}\right.$ : $\mathrm{LGA}_{\mathrm{L}}, \mathrm{LGA}_{\mathrm{W}}, \mathrm{LGA}_{\mathrm{L}+\mathrm{W}}$; $\mathrm{B}$ : cd vs no-cd; C: breastfed vs formular) were analyzed by using analyses of $t$-test and the covariance (ANCOVA) after adjustments for current age and gender.

A p-value of $\leq 0.05$ was defined as statistically significant.

\section{Results}

\section{Whole group}

44 subjects had siblings who were LGA at birth. 31 mothers (38.8\%) and 21 fathers (34.4\%) were born LGA. A positive correlation was found between subject's birth weight SDS and subjects mother's birth weight-SDS ( $\mathrm{r}: 0.23, \mathrm{p}=0.042)$ and mother's birth length ( $\mathrm{r}: 0.25$, $\mathrm{p}=0.027$ ).

Weight and height development: All anthropometric data are shown in Table 1. Weight and height development are shown in Figures 1 and 2. Significant catch-down (negative change $>0.67$ SDS) for weight and for length was observed for the vast majority of the group (88\%) already in the first year of life. Catch-down for weight as well as for length occurred during the first year beginning mostly at three months of age and lasting until one year. Height and weight-SDS remained unchanged thereafter. Significant change of BMI-SDS (BMI difference between two time points: $0.67 ; \mathrm{p}<0.001$ ) occurred during the first months of life and remained unchanged thereafter. Actual mean height-SDS from individuals was significant different from target height (0.22 SDS vs 0.66 SDS; $\mathrm{p}<0.001)$.

Muscle function: For the whole group parameters of muscle function were average for age but below average when corrected for height (data not shown).

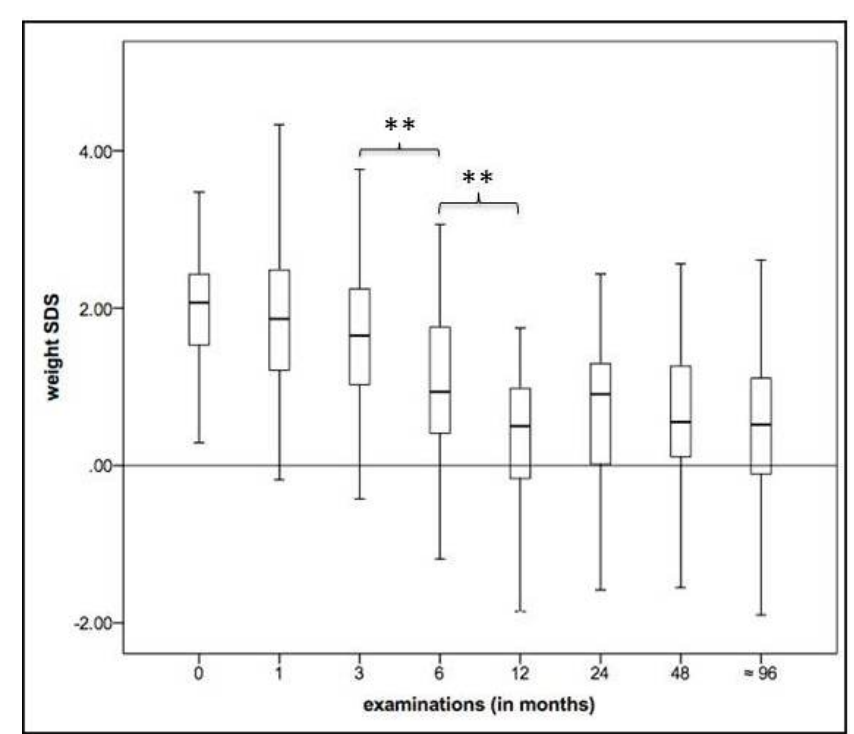

Figure 1: Development of weight-SDS in all formerly LGA-infants from birth to the age of 8 years. Two stars $\left.{ }^{* *}\right)$ indicate a highly significant change between to time points.

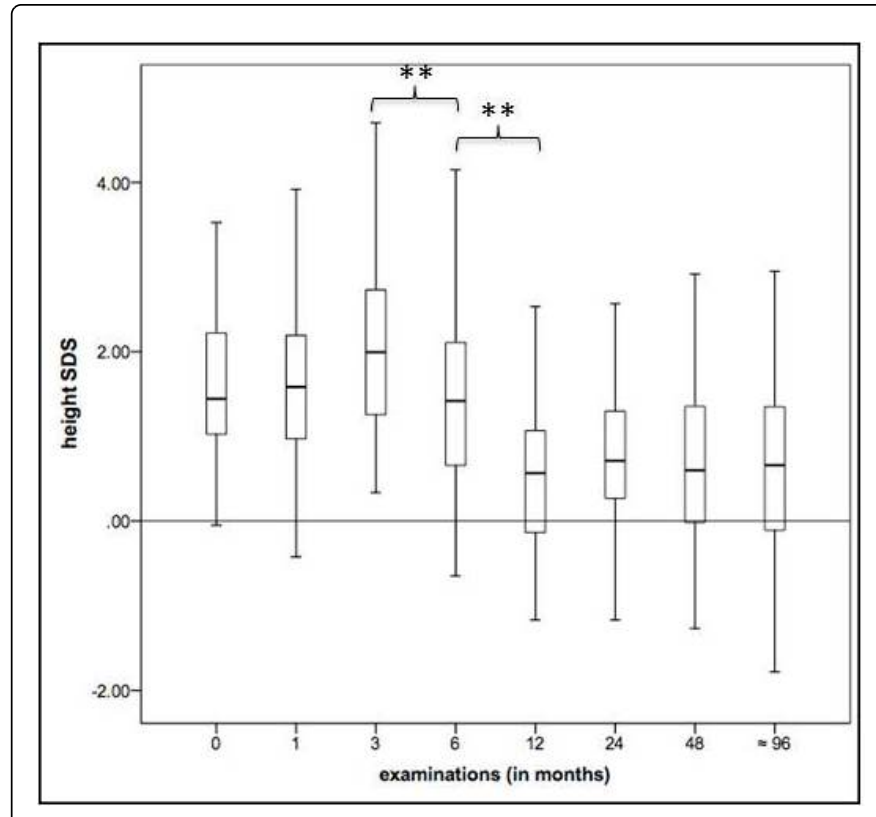

Figure 2: Development of length/height-SDS in all formerly LGAinfants from birth to the age of 8 years. Two stars $\left({ }^{*}\right)$ indicate a highly significant change between to time points.

\section{LGA length vs LGA weight and/or LGA weight+length}

Weight and height development: Irrespective of the classification according to birth parameters all LGA individuals were taller than the average population at the time of the study with no significant difference between the subgroups: Mean height-SDS LGAL 0.86 (SD 1.12), LGAW 0.57 (SD 0.95), LGAW+L 0.86 (SD 1.12). BMI-SDS differed significantly between LGAL (-0.01 SD 1.02) and the LGAL+W group (0.46 SD 0.85; $\mathrm{p}=0.04)$. Differences in other auxological parameters (waist, hip, skin-folds) did not reach statistical significance.

Muscle function: Muscle function differed significantly between the LGA infants who were solely long to those who were classified of LGA due to birth weight. Peak Jump Power according to age and corrected for weight (PJP-SDSweight) was greater in the formerly long versus the heavy LGA-infants: LGAL-0.36 (SD 0.8) vs LGAW+L -0.61 (SD 1.09); $\mathrm{p}=0.04$. Similar results were found by calculation of the Fitness Index: LGAL 98 (SD 12) vs LGAW+L 93 (SD 21); $\mathrm{p}=0.03$. Grip force - SDS and Peak Jump Force (PJF) - SDS did not differ between the subgroups.

\section{Part B: Catch-down growth and body composition}

12 patients (12\%) of the whole group did not catch-down in accordance to the definition of a negative change of weight-SDS of more than 0.67 SDS.

Individuals who did not catch-down were still significantly taller $(+1.57$ SDS \pm 0.85 vs 0.54 SDS $\pm 1.0 ; \mathrm{p}=0.001)$ and heavier than those who showed a catch-down (weight-SDS $(+0.99 \pm 0.59$ vs $0.45 \pm 1.0$; $\mathrm{p}=0.013$ ). Therefore, no difference in BMI-SDS was found. Both groups had no difference in mean target height.

However, by comparing the quartile with highest catch-down (c-d $\max$ ) to those without or very moderate catch-down (c-d low) (Figure 3) we found that the individuals with no catch-down had more total 
Citation: Peters JT, Woelfle J, Joergens S, Schreiner F, Bartmann P, et al. (2017) Effect of Post-Natal Catch-Down and Feeding Practices on Auxology, Body Composition and Muscle Function in Children Born Large-For-Gestational-Age. J Diabetes Metab 8: 760 . doi: $10.4172 / 2155-6156.1000760$

Page 4 of 6

subcutaneous fat mass than the individuals with high catch-down (skin fold SDS difference: 1.1; $\mathrm{p}=0.007$ ). Significant deviations were identified between these two groups in waist and hip circumference SDS (waist circumference SDS difference: -0.6; $\mathrm{p}=0.025$; hip circumference SDS difference: $-0.6: \mathrm{p}=0.04$ ).

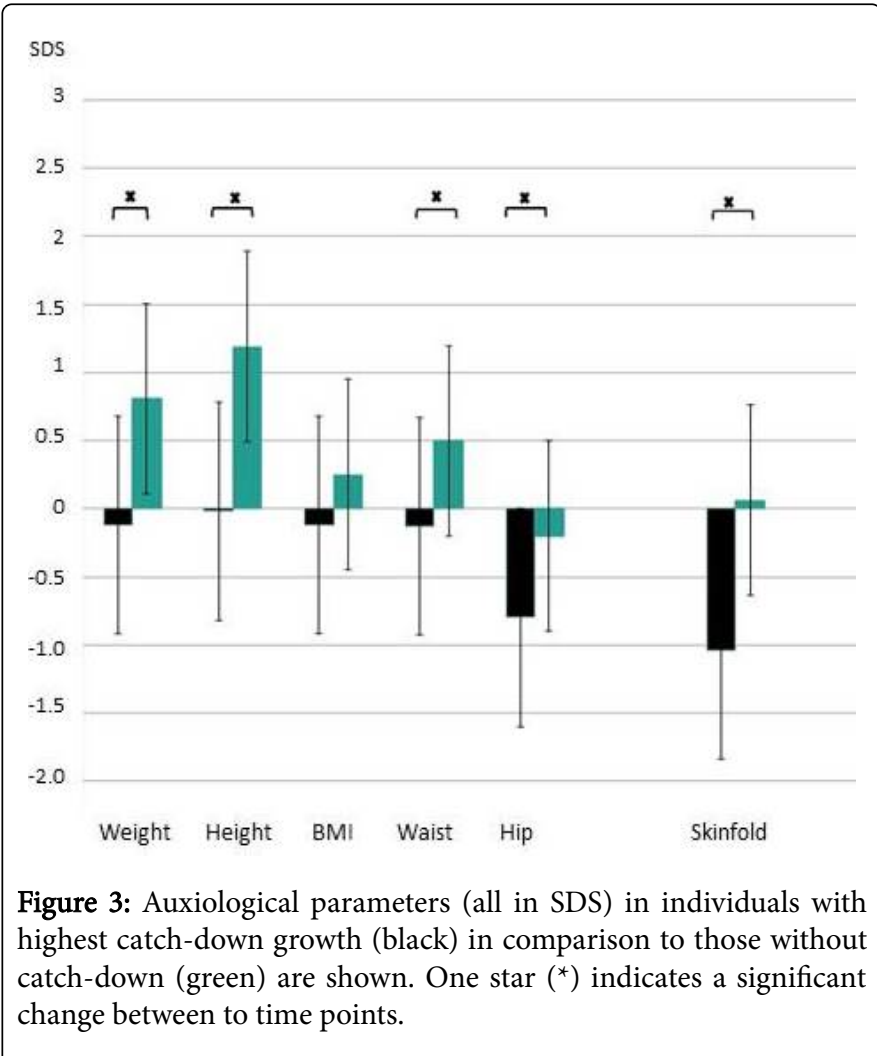

\section{Part C: feeding practices}

No statistical differences for actual auxological or body composition parameters were found between the 60 infants who were exclusively breastfed and those 41 individuals who received additional formula feeding (weight-SDS 0.22 (SD 0.95) vs 0.31 (SD 0.89); BMI-SDS 0.49 (SD 0.89) vs 0.56 (SD 1.01). Out of the 12 who did not show postnatal catch-down growth six (50\%) were exclusively breastfed.

Growth development did not correlate with feeding practice, i.e. breastfeeding or formular supplementation. But post-natal catch-down growth was significantly associated with height-SDS $(r=0.67 \mathrm{p}=0.006)$ and BMI-SDS $(\mathrm{r}=0.33 ; \mathrm{p}=0.04)$ at 8 years.

\section{Discussion}

Present analyses revealed some major findings: By excluding a maternal gestational pathology (gestational diabetes) as a reason for LGA we found a high familiarity in our study group. Only postnatal catch-down growth itself - irrespective of feeding practice - was associated with actual height-, weight-, and BMI-SDS. No implications of feeding practices on parameters of body composition were found. However, although the vast majority of studied LGA children showed a catch-down growth during the first year of life, this did not lead towards a complete normalization of their auxological parameters: Height remained above average and significantly different from target height. In addition, we found distinct differences whether the classification "LGA" was through birth weight and/or birth length-SDS, which underlines the importance for this differentiation.

\section{Family}

In the present data we found a correlation between mother's birth weight and subject's birth parameters. These results correspond with two Swedish studies in 2006 [24] and 2012 [25]. The retrospective epidemiologic study by Ahlsson et al. [24] depict that females born LGA have an increased risk giving birth to an offspring born LGA. Moreover, the risk increases if mothers are overweight or obese. Cnattingius et al. [25] confirm that the risk of an LGA offspring was highest among the women with the highest BMI. Interestingly, no information is given about paternal data. The present data did not show a statistical significant paternal influence on birth weight either, although $20 \%$ of the fathers were LGA at birth.

\section{Height and weight development, catch-down growth}

The present study revealed that LGA-children start their catchdown growth as early as three months of age. This catch-down lasts until the end of the first year of life. However, there was no complete normalization - LGA children remained taller and heavier than the average population and differed significant from target height at the age of eight years. From studies in small-for-gestational-age children it is known that catch-up growth is associated with an increased risk for obesity and metabolic disturbances later in life [4]. Therefore it was assumed that a "mirroring" effect could be observed in LGA infants in which those LGA with the most pronounced catch-down growth might be protected from metabolic disturbances [13] which are associate with LGA birth parameters in general [9]. Taal et al. [11] showed that subjects born LGA without catch-down growth had more subcutaneous fat mass (central and peripheral) and had higher BMI. They conclude that children born LGA had an increased risk of childhood overweight and catch-down might be protective. In confirmation, our study shows that those LGA-children with catchdown growth were smaller and had less central and peripheral fat than those with none or only little catch-down growth. Thus our findings support the hypothesis that catch-down in LGA might have a protective influence on body composition.

Several studies as well as meta-analyses demonstrated at least a modest association between breastfeeding and reduction of the risk of obesity in infants with normal birth-weight $[14,15]$. However, this might be different in the special group of LGA-infants. In accordance with a recent study [16] we found that it seems to be the postnatal catch-down growth itself - irrespective of mode of nutrition - is important to achieve a normalization of auxological parameters and body composition. However, further multicenter studies with populations of children without being LGA are required.

The hypothesized difference of muscle strength in relation to catchdown growth was not confirmed. However, only 12 individuals did not catch-down and results for muscular function had a wide variability. Therefore, studies including higher numbers of patients are needed to rule out or support an effect of catch-down growth on muscular function.

\section{Definition of LGA through birth-weight versus birth-length}

In the current study we assumed to find differences in children born large for gestational age due to increased birth weight in contrast to 
Citation: Peters JT, Woelfle J, Joergens S, Schreiner F, Bartmann P, et al. (2017) Effect of Post-Natal Catch-Down and Feeding Practices on Auxology, Body Composition and Muscle Function in Children Born Large-For-Gestational-Age. J Diabetes Metab 8: 760. doi: $10.4172 / 2155-6156.1000760$

Page 5 of 6

those with solely increased birth length. Although we found that all infants born LGA were still tall at 8 years and still taller than target height, those who have been "only" long at birth (LGAL) presented with a complete normalization of their BMI whereas those with heavy birth weight had a BMI nearly $0.5 \mathrm{SD}$ above the mean which differed significantly from the LGAL. Xie et al [26] showed that LGA-infants with co-occurrence of maternal overweight/obesity and diabetes had the greatest 4-year BMI. They defined "a healthy LGA-phenotype" if there was neither maternal diabetes overweight/obesity nor excessive gestational weight gain. In our study we selected only LGA-infants from mothers without diabetes and/or gestational diabetes because we aimed to find differences of growth and weight development in accordance to birth parameters i.e. whether the newborn was solely long and/or heavy. Similar to those who were born LGA due to maternal gestational diabetes we found that those infants born heavy and long due to other reasons had continuous higher rising BMI than those born LGA only defined by birth length. The later could be defined as the "healthy phenotype"-especially by taking into account that we found a strong familiarity.

Weight and BMI do not necessarily reflect lean or fat mass. The few studies, which analyzed lean body mass by using direct methods like DEXA or body plethysmography showed increased fat mass in LGA infants in comparison to AGA infants [27,28]. Muscle mass and muscle metabolism are important determinants of insulin sensitivity and glucose tolerance $[28,29]$. In the present study in addition to the assessment of grip force we used the analysis of two-leg jump as a parameter for the power of the muscles, and the muscular coordination as well as the effectiveness of the movement. We found all parameters of muscular function below average when corrected for weight. In addition, a significant difference was found for the multijoint movement requiring the coordination of many muscles between those born only long $\left(\mathrm{LGA}_{\mathrm{L}}\right)$ in contrast to those who have been born heavy $\left(\mathrm{LGA}_{\mathrm{W} / \mathrm{W}+\mathrm{L}}\right)$. Peak jump power was significant better in those long at birth than the heavy ones. And this difference remained after correcting for actual weight. Therefore, present data underline the importance of differentiation between birth-length and birth-weight!

\section{Conclusion}

Irrespective of feeding practice the majority of LGA-children showed catch-down growth. However catch-down growth did not lead to a complete normalization of auxologic parameters. Importantly in those without postnatal catch-down growth higher indices for fatness were observed. Additionally a higher BMI-SDS and worse muscular function at the age of eight years were observed in relation to whether the former newborn had been super normally heavy or just long. This underlines the importance for a distinction whether birth weight or birth length lead to the classification of "large-for-gestational-age". It confirms that those who are too heavy at birth might be at increased risk to develop obesity which can be reduced by achieving a postnatal catch-down growth irrespective of the mode of nutrition.

\section{Declarations}

\section{Ethics approval and consent to participate}

The Ethics Committee of the University Hospital Bonn reviewed the study. Subjects and parents gave their informed consent.

\section{Consent for publication}

Not applicable

\section{Availability of data and material}

The datasets used and/or analysed during the current study available from the corresponding author on reasonable request.

\section{Competing interests}

The authors declare that they have no competing interests.

\section{Funding}

There was no funding for this study.

\section{Authors' contributions}

All authors (JT-P, JW, SJ, FS, PB, BG have made substantial contributions to conception and design, acquisition of data, and analysis and interpretation of data. JT-P has written the initial article, which was critically revised by all others. All have given their final approval of the version to be published.

JT-P conceptualized and designed the study, examined the patients, collected all data, drafted the initial manuscript, and approved the final manuscript as submitted.

JW carried out the initial analyses, helped with analysis and interpretation of the data. He reviewed and revised the manuscript, and approved the final manuscript as submitted.

SJ designed the data collection instruments, and coordinated and supervised data collection. She examined the patients together with JTP.

FS designed the data collection instruments, reviewed and revised the manuscript, and approved the final manuscript as submitted.

$\mathrm{PB}$ conceptualized and designed the study reviewed and helped with analysis and interpretation of the data. He revised the manuscript, and approved the final manuscript as submitted.

BG conceptualized and designed the study, carried out the initial analyses and helped with analysis and interpretation of the data. She reviewed and revised the manuscript, and approved the final manuscript as submitted.

\section{References}

1. Sebastiani G, Díaz M, Bassols J, Aragonés G, López-Bermejo A, et al. (2016) The sequence of prenatal growth restraint and post-natal catch-up growth leads to a thicker intima-media and more pre-peritoneal and hepatic fat by age 3-6 years. Pediatr Obes 11: 251-257.

2. Barker DJP (1997) Fetal nutrition and cardiovascular disease in later life. Br Med J 53: 96-108.

3. Carmudan MO, Carmudan AD, Polat S, Beyazova U (2011) Growth Patterns of large, small, and appropriate for gestational age infants: impacts of long- term breastfeeding: a retrospective cohort study. J Pedriatr Endocr Met 24: 463-468.

4. Ong KL, Ahmed ML, Emmett PM, Preece MA, Dunger DB, et al. (2000) Association between postnatal catch-up growth and obesity in childhood: prospective cohort study. Br Med J 320: 967-971.

5. Dyer JS, Rosenfeld CR, Rice J, Rice M, Hardin DS (2007) Insulin resistance in Hispanic large-for-gestational-age neonates at birth. J Clin Endocrinol Metab 92: 3836-3843. 
Citation: Peters JT, Woelfle J, Joergens S, Schreiner F, Bartmann P, et al. (2017) Effect of Post-Natal Catch-Down and Feeding Practices on Auxology, Body Composition and Muscle Function in Children Born Large-For-Gestational-Age. J Diabetes Metab 8: 760 . doi: $10.4172 / 2155-6156.1000760$

Page 6 of 6

6. Evagelidou EN, Giapros VI, Challa AS, Cholevas VK, Vartholomatos GA, et al. (2010) Prothrombotic state, cardiovascular, and metabolic syndrome risk factors in prepubertal children born large for gestational age. Diabetes Care 33: 2468-2470.

7. Hediger ML, Overpeck MD, Kuczmarski RJ, McGlynn A, Maurer KR, et al. (1998) Muscularity and fatness of infants and young children born small or large-for-gestational-age. Pediatrics 102: E60.

8. Hediger ML, Overpeck MD, McGlynn A, Kuczmarski RJ, Maurer KR, et al. (1999) Growth and Fatness at Three to Six Years of Age of Children Born Small or Large-For-Gestational Age. Pedriatrics 104: e33.

9. Johnsson JW, Haglund B, Ahlsson F, Gustafsson J (2015) A high birth weight is associated with increased risk of type 2 diabetes and obesity. Pediatr Obes 10: 77-83.

10. Toschke AM, Grote V, Koletzko B, von Kries R (2004) Identifying children at high risk for overweight at school entry by weight gain during the first 2 years. Arch Pediatr Adolesc Med 158: 449-452.

11. Taal HR, van Heijden AJ, Steegers EAP, Hofmann A, Jaddoe WV (2013) Small and large for gestational age at birth, infant growth, and childhood overweight. Obesity 21: 1261-1268.

12. Chiavaroli V, Derraik JGB, Hofman PL, Cutfield WS (2015) Born Large for Gestational Age: Bigger Is Not Always Better. J Pediatr 17: 1-5.

13. Fricke O, Weidler J, Tutlewski B, Schoenau E (2006) Mechanography- A new Device for the Assessment of muscle function in pediatrics. Pediatr Res 59: 46-44.

14. Arenz S, Rückerl R, Koletzko B, von Kries R (2004) Breast-feeding and childhood obesity--a systematic review. Int J Obes Relat Metab Disord. 28: $1247-1256$.

15. Harder T, Bergmann R, Kallischnigg G, Plagemann A (2005) Duration of breastfeeding and risk of overweight: a meta-analysis. Am J Epidemiol 162: 397-403.

16. Salahuddin M, Pérez A, Ranjit N, Hoelscher DM, Kelder SH (2017) The associations of large-for-gestational-age and infant feeding practices with children's body mass index z-score trajectories: the Early Childhood Longitudinal Study, Birth Cohort. Clin Obes.

17. Voigt M, Schneider KT, Jährig K (1996) Analysis of a 1992 birth sample in Germany: new percentile values of the body weight of newborn infants (German). Geburtshilfe Frauenheilkd 6: 550-558.

18. Xu H, Simonet F, Luo ZC (2010) Optimal birth weight percentile cut-offs in defining small- or large-for-gestational-age. Acta Paediatr 99: 550-555.
19. Rauch F, Neu CM, Wassmer G, Beck B, Rieger-Wettengel G, et al. (2002) Muscle analysis by measurement of maximal isometric grip force: new reference data and clinical applications in pediatrics. Pedriatr Res 51: 505-510.

20. Fricke O, Roedder D, Kribs A, Tutlewski B, von Kleist- Retzow JC, et al. (2010) Relationship of muscle function to auxology in Preterm Born Children at the Age of Seven Years. Horm Res Paediatr 73: 390-397.

21. Kromeyer-Hauschild K, Wabitsch M, Kunze D, Geller F, Geiß HC, et al. (2001) Perzentile für den body mass index für das Kindes- und Jugendalter unter Heranziehung verschiedener deutscher Stichproben (German). Monatsschr Kinderheilkd. 149: 807-818.

22. Rosario AS, Schienkiewitz A, Neuhauser H (2011) German height references for children aged 0 to under 18 years compared to WHO and CDC growth charts. Ann Hum Biol 38: 121-130.

23. Fernández JR, Redden DT, Pietrobelli A, Allison DB (2004) Waist circumference percentiles in nationally representative samples of AfricanAmerican, European-American, and Mexican-American children and adolescents. J Pediatr 145: 439.

24. Ahlsson F, Gustafsson J, Tuvemo T, Lundgren M (2007) Females born large for gestational age have a doubled risk of giving birth to large for gestational age infants. Acta Paediatr 96: 358-362.

25. Cnattingius S, Villamor E, Lagerros YT, Wikström AK, Granath F (2012) High birth weight and obesity-a vicious circle across generations. Int J Obes (Lond) 36: 1320-1324.

26. Xie C, Wang Y, Li X, Wen X (2016) Childhood Growth Trajectories of Etiological Subgroups of Large for Gestational Age Newborns. J Pediatr 170: 60-66.

27. Hammami M, Walters JC, Hockman EM, Koo WW (2001) Disproportionate alterations in body composition of large for gestational age neonates. J Pediatr 138: 17-21.

28. Ma G, Yao M, Liu Y, Lin A, Zou H, et al (2004) Validation of a new pediatric air-displacement plethysmograph for assessing body composition in infants. Am J Clin Nutr 79: 653-660.

29. Tresierras MA, Balady GJ (2009) Resistance training in the treatment of diabetes and obesity: mechanisms and outcomes. J Cardiopulm Rehabil Prev 29: 67-75. 